Ocean Engng. Vol. 7, pp. 281-304.

Pergamon Press Ltd. 1980. Printed in Great Britain

\title{
FULL SCALE WAKE AND BOUNDARY LAYER INSTRUMENTATION FEASIBILITY STUDY
}

\author{
Vernon A. Phelps \\ Research Scientist and Project Director \\ Armin W. Troesch \\ Assistant Research Scientist \\ and \\ JOHN P. HACKETT \\ Graduate Research Assistant
}

Department of Naval Architecture and Marine Engineering, College of Engineering, The University of Michigan, Ann Arbor, Michigan 48109, U.S.A.

\begin{abstract}
Almtract-Fluid-flow measuring devices intended for full scele use were designed and tested. One dovice coneisted of a rake supporting different types of pitot tubes. This will be used to obeain a velocity prodile of the boundary layer of a test ship. The other devioe was a five-hole pitot tube that was unod in a wake survey. The pressure transport mutham (i.e. the modium that carried the preseure simal from the pitot tube to the prowoure tranducer) was itr indtead of water. This proved to be successful, as the results indicate.
\end{abstract}

\section{INTRODUCTION}

Propellers for high speed ships are designed on the basis of information obtained from model tests. The model speed is scaled according to Froude's law, but the boundary layer, which influences the flow into the propeller, follows a Reynold's scaling.

A number of recent papers have investigated the problems of full-scale wake prediction when these scaling effects are considered. Yokoo (1974) did a survey of the available literature and concluded that there are still unknown factors in the prediction of the wake pattern based on model tests. Since the character of the boundary layer in front of the propeller influences the flow into the propeller, it is important to understand what scaling effects take place. Taniguchi and Fujita (1970) show that not only does the magnitude of the velocities in the boundary layer change from model to full-scale, but so does the direction of the velocity components. This has an appreciable effect on the propeller race as shown by Namimatsu et al. (1973). They compare the estimated flow patterns of a 200,000 D.W.T. tanker based on model tests with the results from full-scale tests. In a recent work by Canham (1974), comparisons between model tests and full-scale tests of a Leander Class frigate (HMS Penelope) were made. The author concludes there is "no significant wake scale effect in this type of frigate". However, this conclusion may be drawn from the data only if the mean wake fraction is considered. There were significant differences in local velocities in the propeller race which were unexplained. When Yokoo (1974) reviewed Canham's paper, he speculated that the supposed lack of scale effect was a result of the 
increase in ship wake due to surface roughness. This would increase the boundary layer of the ship, making it more similar to that of the model and thus decreasing the differences between model and ship velocities in the propeller race.

These papers agree in that they point out a major difficulty in current propellet design procedures, i.e. accounting for the scale effects on the wake when going from model to full scale.

The objective of the work described in this paper was to design and develop a flow meter capable of determining the wake and boundary layer character in the vicinity of the propeller of full-scale test vessels. The instrument package was chosen in such a manner that the system would produce stable and reliable data over the required test range. The emphasis of the design was on reliability, and how easily a component could be replaced.

After a search of the literature, it was decided to use pitot tubes to find the velocity profile. The reliability of the pitot tube relative to other flow measurement devices such as blade wheel speed meters, ultra-sonic meters, hot film meters, and laser doppler meters has been demonstrated by Yokoo (1974). However, there are a number of problems associated with pitot tubes and in this respect an alternative method of using them is described. In most previous cases when pitot tubes were used for full-scale testing, water was used as a transport medium. That is, the tubes carrying the pressure signals from the head of the pitot tubes to the pressure transducers were filled with water. Should air become entrapped in the lines, the calibration of the instrument would be invalidated. A method by which the water is replaced with air is described in the following sections.

The boundary layer measurement devices included two types of pitot tubes mounted on a rake. One of these was a total head tube and the other was a Prandtl-type pitot-static tube. For the measurements in the region of the propeller wake, a five-hole pitot tube is proposed. These are described in detail in the following sections.

\section{THE SELECTION OF AIR OR WATER AS A TRANSPORT MEDIUM}

One of the anticipated problems with the system was the method by which pressures on the actual measuring devices, i.e. the total head tube, the Prandtl-type pitot tube and the spherical head pitot tube, would be recorded for analysis. When these types of instruments are used in a laboratory environment, it has been common practice to use the fluid whose velocities and piessures are being measured as the medium that transmits the pressure from the head of the tube to a pressure transducer. For example, in a wind tunnel a pitot tube may be connected to a mercury manometer by a flexible hose with air in it, and in a towing tank the same pitot tube may be connected to a manometer or electronic pressure transducer by a hose filled with water. When these same instruments are scaled up for use on a full-scale application, some minor laboratory problems become magnified.

Since the primary purpose of the instrument package being developed was to measure the wake velocities behind an actual ship, a natural choice would have been to use salt water as the transport medium. However, most of the transducer manufacturers' catalogs carefully spell out the undesirability or impossibility of using many types of instrumentation in corrosive fluids, such as salt water. One possible approach to the solution of this problem would be to use distilled water in the lines. However, the use of any heavy fluid, i.e. water, oil, etc., has a major drawback in that air bubbles tend to collect in the lines. These air bubbles reduce the accuracy of the system substantially. In a laboratory it is possible to remove the bubbles from the lines when they are relatively short. However, when the 
equipment is used at sea, the pressure lines will be at least $50 \mathrm{ft}$ in length. Namimatsu and Maraoka (1974) experienced this problem. They substituted deaerated water for potable water and found that they got better results. However, one difficulty with deaerated water is that it is not possible tc remove all the air completely. If the transducer is located more than $15 \mathrm{ft}$ above the surface of the water, the partial vacuum in the line will tend to draw out of the water any air missed in the deagration process.

A method described by Takahashi et al. (1970) shows that filling the lines with air by a method called the air-blow system eliminates these problems. Briefly, the air-blow system uses air as the pressure transport medium. Before a reading is taken, a valve which blows all of the water out of the system is opened. The valve is then closed and a measurement is taken. Potential problems with the air-blow system would include a slow system response or even undesirable dynamic effects, the magnitude of the effect of the one air-water interface, and the size and length of the tubing used. Since this method showed promise in view of its simplicity and reliability, it was investigated for possible implementation into the instrument package.

To test the system response, the pressure transducer was connected to one of the pitot tubes and the electronic signal was displayed on an oscilloscope. The pitot tube was placed in the water and run down the tank at different speeds. In addition, the length of the tube was varied from $10 \mathrm{ft}(3.05 \mathrm{~m})$ to $146 \mathrm{ft}(44.5 \mathrm{~m})$. The type of tube used was $1 / 8 \mathrm{in}$. $(3.17 \mathrm{~mm})$ inside diameter Tygon plastic tubing. A typical record from the oscilloscope is shown in Fig. 1. The record represents the pressure measurement from the time the blow pressure was stopped to the time it reached a steady value indicating the pressure at the pitot tube.

The response time, $t$, seemed to be independent of the velocity of the fluid at the pitot tube. For the shorter tube length of $10 \mathrm{ft}(3.05 \mathrm{~m}), t$ was under $1 / 10 \mathrm{~s}$. For the longer lengths of about $150 \mathrm{ft}(45.7 \mathrm{~m}), t$ was estimated to be about $3 \mathrm{~s}$. For the proposed length of $50 \mathrm{ft}$ $(15.24 \mathrm{~m}), t$ reached a value of around $6 / 10 \mathrm{~s}$. There are two observations to be made from the figure. Firstly, the time taken for the system to reach steady state is small relative to the time available in the full-scale trials. Secondly, the lack of oscillations in the record indicates that the system has sufficient damping to minimize any dynamic effects due to resonant vibrations.

The effect of the air-water interface was difficult to measure. There is a pressure drop across the surface due to surface tension; this drop is proportional to the surface curvature and increases as the size of the holes decreases. Since the tust results for the total pressure

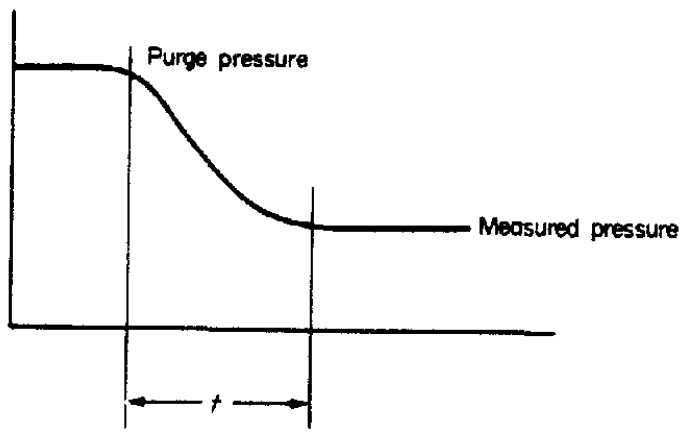

FIG. 1. System response with the air-blow method. 
tube, as described in a later section, compare so well with the carriage speed, the effects of the air-water interface, as applied to the instrument package, will be considered to be small.

Finally there were no noticeable differences encountered when $1 / 16 \mathrm{in}$. $(1.59 \mathrm{~mm})$ i.d. tubing replaced the $1 / 8 \mathrm{in}$. $(3.18 \mathrm{~mm})$ i.d. tubing. We recommend that the larger diameter tubing be used for the part of the lines carrying the flushing air, while the smaller lines may be used for the tubes leading to the pressure transducer. For a more complete description of the test apparatus, see the next section.

\section{MEASURING TECHNIQUES}

At the initial stages of this project, the decision on the transport medium-either air or water-had not yet been made. As a result, the measuring and recording systems were designed to handle either air or water. Whereas it quickly became apparent that the deaerated water process was more complex than the air-blow process, it could not be eliminated until the air-blow process was proven successful. As a result, some unnecessary components were included in the first design of the measuring and recording systems. For a schematic representation of the device, see Fig. 2.

The water reservoir $D$ and the glass tubes $K$ were intended for use in the deacrated-

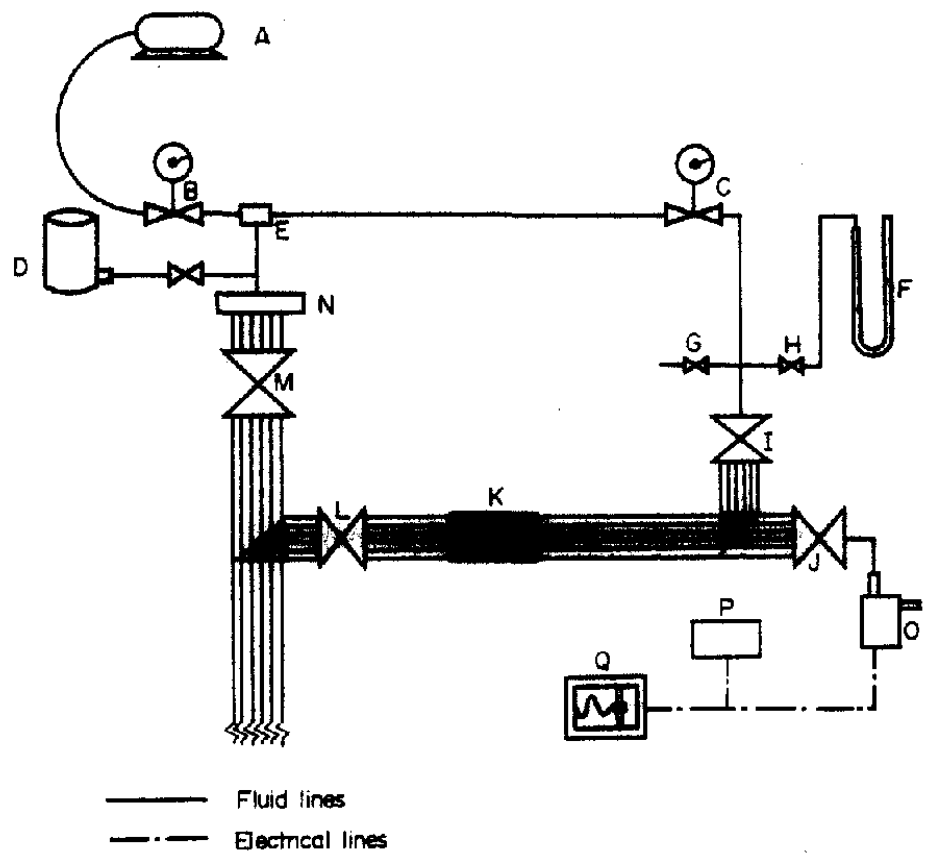

FiG. 2. Genecal arrangoment of measuring apparatus.

A: air compressor and 2.5 gal $(9.46 \mathrm{I})$ tank rated at $100 \mathrm{psi}\left(68.9 \mathrm{~N} / \mathrm{cm}^{2}\right)$ and pumping rate of $2 \mathrm{gal} / \mathrm{min}$ ( $7.57 \mathrm{~V} / \mathrm{min}) ; \mathrm{B}$ and C: regulator valves with pressure gauges; D: aerated water reservoir; E: three-way valve; F: manorneter uing 2.94 specific gravity fluid; $G$ and $H$ : cocks; I and J: Scanivalve, model W0601/1: $12 \mathrm{~T} 12$ input lines, 1 output line; K: glass tubes 1/4-in. (6.35 mm) OD; L and M: Scanivalve, model W1260/6P-1T, 6 input lines 6 output lines (theace valves acted as off-on switches for 6 lines simultaneously); N: manifold; O: two port, differential pressure transducer $\pm 4 \mathrm{psi}\left( \pm 2.76 \mathrm{~N} / \mathrm{cm}^{2}\right) ; \mathrm{P}$ : voltmeter; $\mathrm{Q}: \mathrm{x}-\mathrm{y}$ plotter. 
water-flush system. Once the air-blow system was proven effective, these parts were not used. The manifold $\mathbf{N}$ was necessary, because without it, the lines leading to the pitot tubes would have seen different blow pressures. This could have resulted in some lines not being purged at all.

The manometer $F$ was used to provide a calibration check on the pressure transducer o. In this procedure, the valve at $B$ was partially opened, allowing a pressure of approximately 3 psi $\left(2.07 \mathrm{~N} \mathrm{~cm}^{-2}\right)$ to enter the lines from the air compressor A. The off-on Scanivalves* at $L$ and $M$ were closed. The cock at $G$ was closed and the one to the manometer at $\mathrm{H}$ was opened. The Scanivalves at $\mathrm{I}$ and $\mathrm{J}$ were set on the same tube number allowing the pressure measured by the manometer to be measured also by the pressure transducer. The pressure acting on the manometer and pressure transducer could be varied by the regulator valve $\mathrm{C}$. A calibration curve was thus produced for the pressure transducer. The transducer was of the differential type with two ports. One port was left open to the atmosphere, giving the transducer a range of $\pm 4 \mathrm{psi}\left( \pm 2.76 \mathrm{~N} \mathrm{~cm}^{-2}\right)$ gauge pressure. To zero the transducer, the valve at $C$ was closed and the cock at $G$ was opened. This vented the lines connecting the manometer and pressure transducer to the atmosphere, giving the transducer a zero differential.

An actual test run was conducted in the following manner. First the valve at $C$ would be closed and the Scanivalve at I would be set on a line number that had been capped. The other 12/1 Scanivalve at $J$ would be set on the first line to be measured. The Scanivalve at $L$ would then be set on the open position. As the towing carriage accelerated up to the desired speed, the Scanivalve at $M$ would remain open, purging with air all lines that were to be measured. Once the test speed had been achieved, the Scanivalve at $M$ was closed, stopping the purging pressure and allowing the total pressure head at the pitot tube to be measured. The Scanivalve at $J$ was then quickly set at the next line number to be measured and the purge pressure was again allowed in the lines by opening the Scanivalve M. After a quick blow, this valve was closed and a pressure measurement was recorded. This procedure of switch-purge-read was then repeated until all the lines had been sampled or the end of the towing tank had been reached. We found that purging a line for a fraction of a second was sufficient to remove any water that may have been in the line. For an example of the recorded output from a test run of the five hole pitot tube, see Fig. 3. Note that the different line pressures can be easily separated as a result of the distinctive peaks in the plot due to the purge pressure. The chart rate was $2.5 \mathrm{~s} \mathrm{~cm}^{-1}$.

The ease with which the numerous measurements could be made was impressive. Having only one pressure transducer reduced the amount of time needed for electronic balancing and calibration. The regulator and pressure gauge at $C$ were redundant and found to be unnecessary. The Scanivalves were hand operated which resulted in some blow times being too long and some pitot-pressure measurements being too short. Driving these valves by an electric motor should remove this difficulty.

\section{BOUNDARY LAYER MEASUREMENTS}

Boundary layer rake

The first application of the boundary layer measurement devices will be to obtain a boundary layer velocity distribution on the hull bottom of a research vessel along the after-section of the hull. Initial estimates of the expected boundary layer thickness there

-Scanivalve is the trade name of a device that acts as a pressure multiplexer. 


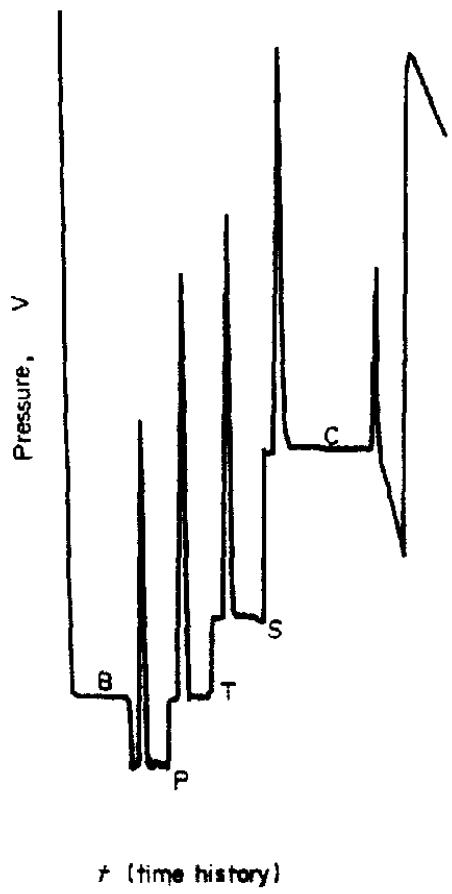

$$
\begin{aligned}
& \text { Symbols for fivethole pitot tube: } \\
& \text { B-bottom hole } \\
& P \text { - port hole } \\
& T \text { - top hole } \\
& S \text { - starboord hole } \\
& C \text { - center hole }
\end{aligned}
$$

FiG. 3. Example of recorded pressure output for a five-hole pitot tube.

were less than a foot, hence a boundary layer rake of 12 in. was selected. The gradient of the boundary layer velocity distribution is greatest near the hull. This then should be the region of the largest concentration of the probes. A total of ten probes were installed in the rake, eight total pressure pitot tubes and two pitot-static tubes. Figure 4 shows the rake with probes installed (see Fig. 23 at the end of this report for a detailed drawing). Tube locations on the rake were labeled starting with the probe nearest the hull as A to the outermost probe as J. Placement of one pitot-static tube at the outermost rake position tended to minimize the interference effects, as will be discussed later. The pitot-static tubes will be used to determine the static head and the flow direction, and how each of these changed within the boundary layer.

\section{Total head pitot tube}

The use of air as the flushing medium and also the pressure transmission medium in a water environment meant an air-water interface would exist in the pitot tube. This vertical interface was expected to be at the probe tip. At the interface of any two fluids one must contend with the phenomenon of surface tension. Due to surface tension there is a pressure 


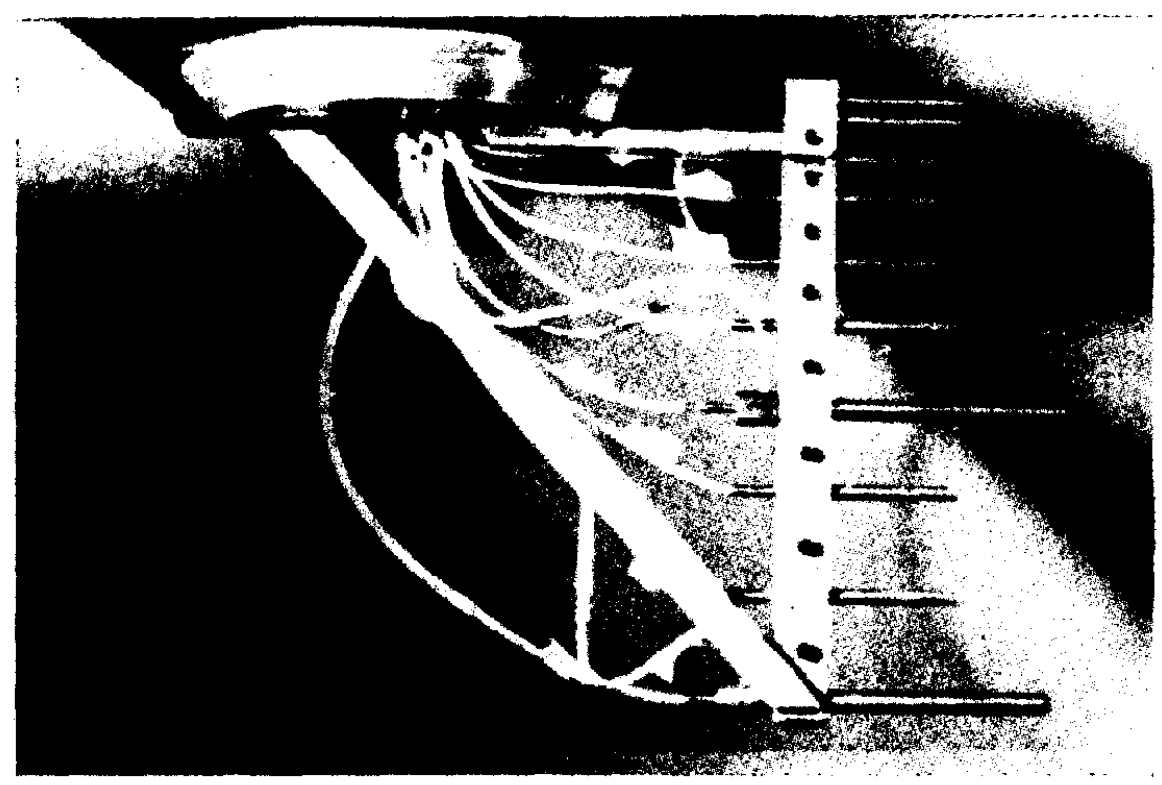

FIG. 4. Pitot tubes with rake. 


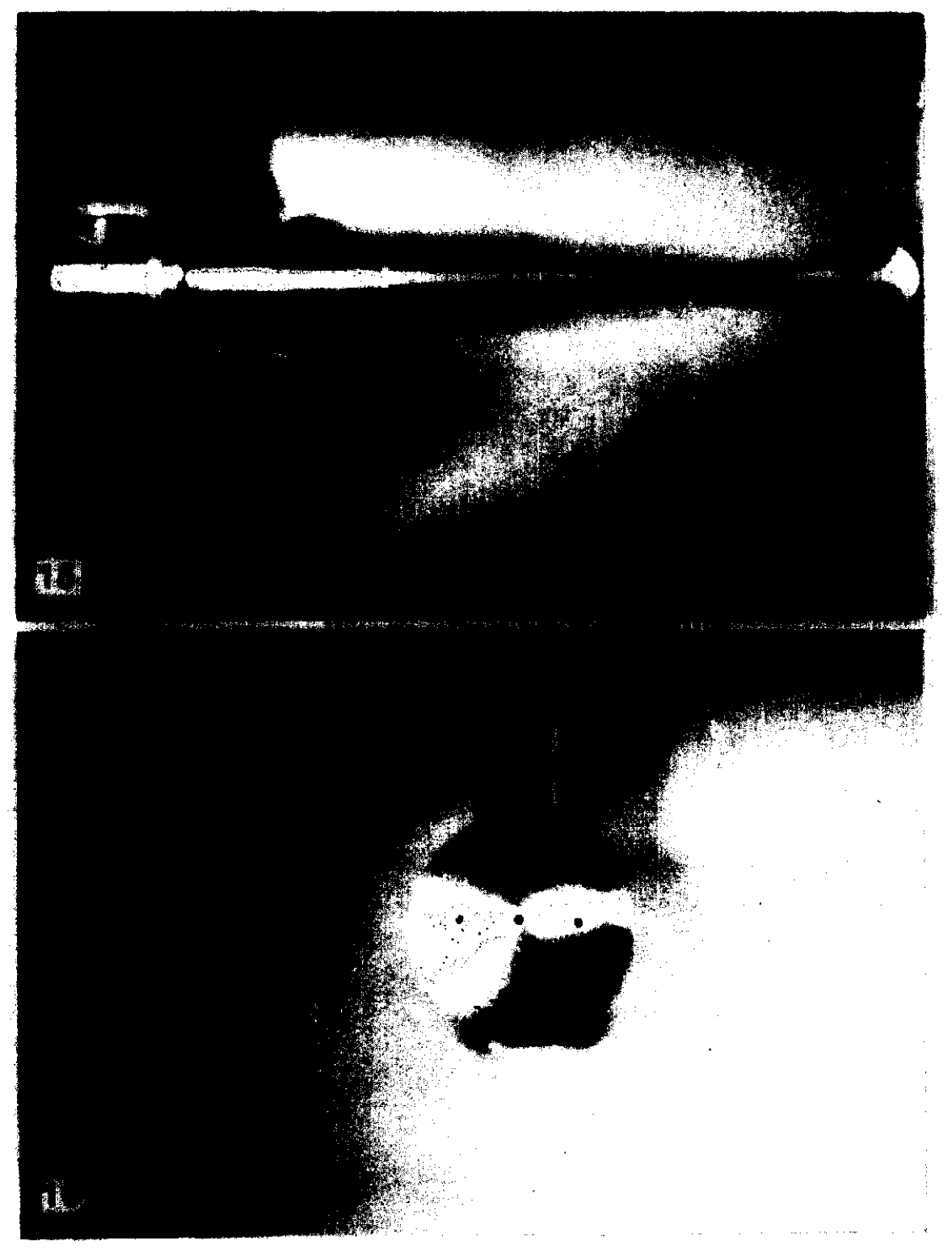

Fra. 15. Five-hole pitot tube and mounting assembly.

FIG, 16. Placement of holes for five-hole pitot tube. 


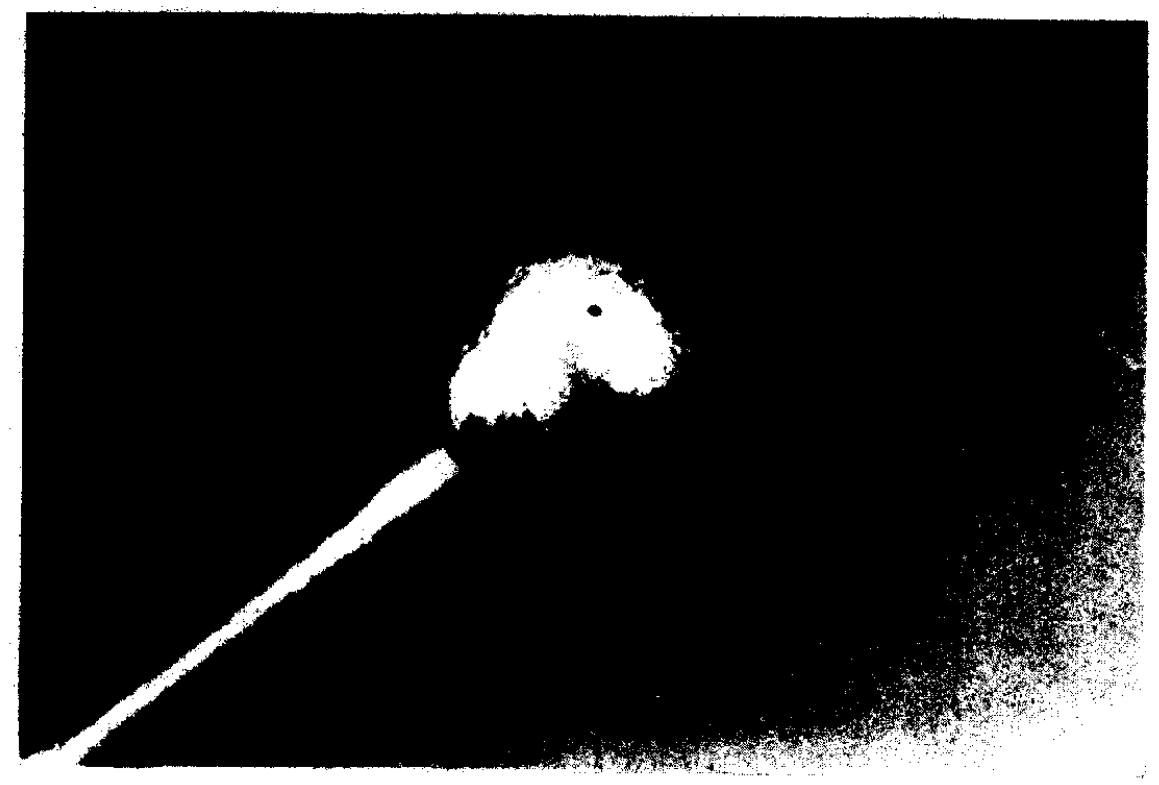

FIG. 18. Five-hole pitot tube with sand added. 


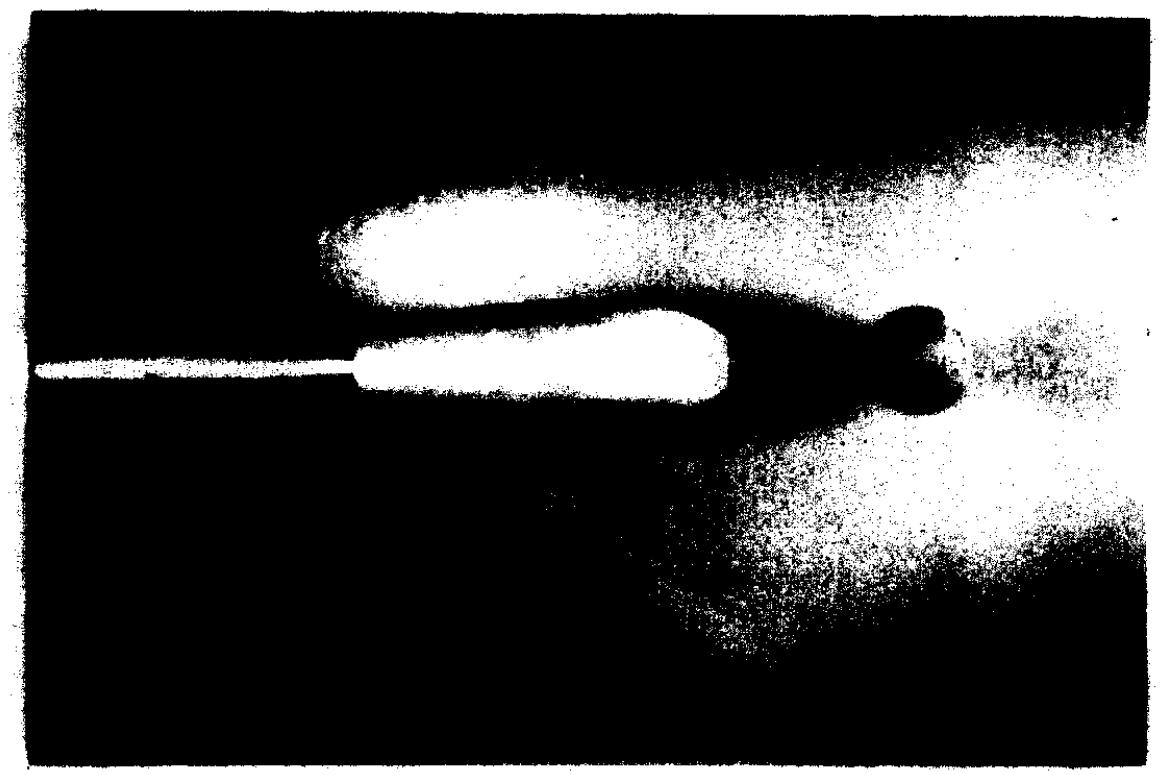

FIG. 21. Five-hole pitot tube with fairing added. 
difference between the water side and the air side of the film. The magnitude of the surface tension (and hence pressure difference) varies in inverse proportion to the tube diameter. If the tube is very small in diameter, the effect of surface tension must be taken into consideration during data analysis. If the tube is fairly large in diameter, the effect of surface tension is small and may be ignored. With the latter goal in mind, our tubes were a reasonably large $1 / 4 \mathrm{in}$. (6.35 $\mathrm{mm}$ ) diameter.

Other reasons for large diameter tubes are the reduced likelihood of the tube clogging due to small marine life and marine pollution and their greater strength, which minimizes the chance of damage during rake installation and test operations. Too large a diameter tube would result in an average pressure reading over a large region of the boundary layer, and large flow perturbations which may influence the readings of neighboring probes.

Therefore the probe design selected here is similar to the apparently successful Japanese design described by the Executive Committee for the Project of Ship Boundary Layer Measurements (1971).

Tube performance. The tubes gave excellent static readings while motionless. The effect of forward speed after subtracting the static head can be seen in Fig. 5. Figure 6, the effect

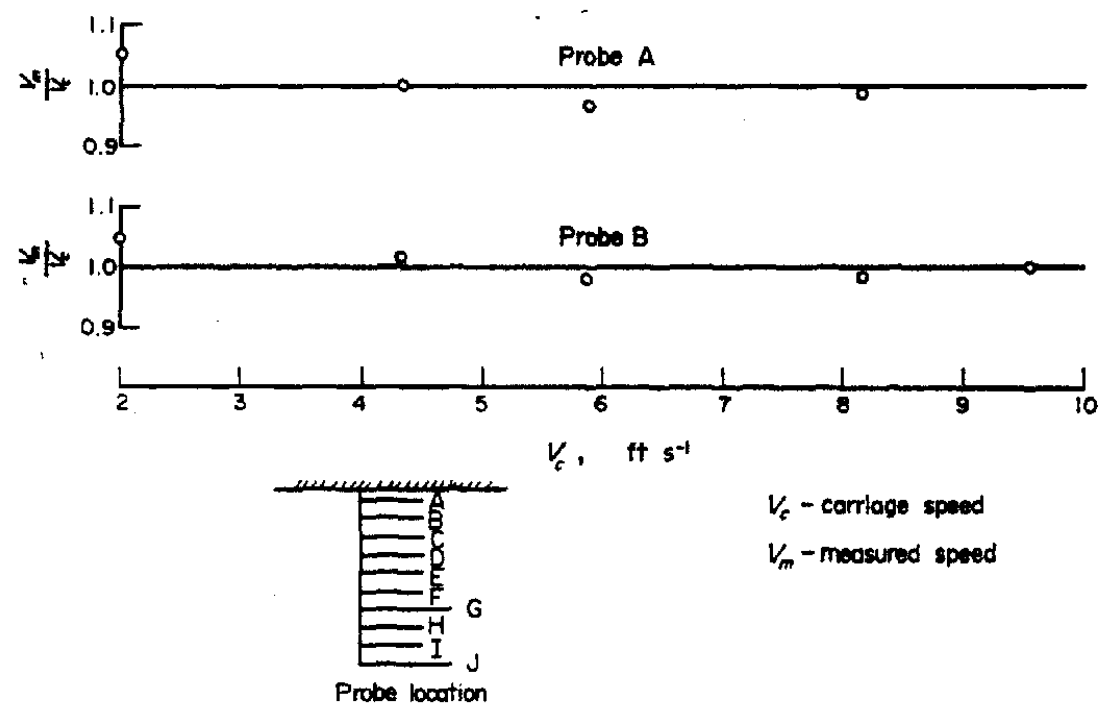

Fio. 5. Total head pitot tube calibration.

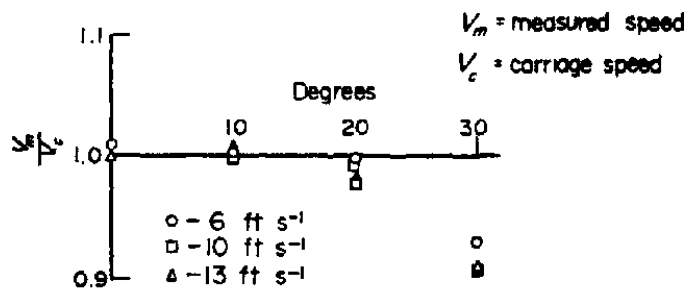

Fic. 6. The effect of flow angle on the total head tube velocity measurements. 
of the angle of the incident flow, shows the total head is insensitive to flow angles up to $10^{\circ}$. At flow angles of $20^{\circ}$ and speeds of $10 \mathrm{ft} \mathrm{s}^{-1}\left(3.05 \mathrm{~m} \mathrm{~s}^{-1}\right)$ or greater, the error is below $2 \%$. We therefore expect good performance from the total head pitot tubes when at sea. This, of course, depends on our ability to determine the static head accurately.

\section{Pitot-static tube}

The classic pitot-static tubes such as the Prandtl tube are known for their ability to give flow speed directly without correction for flow angle at flow angles of up to $15^{\circ}$. These probes can supply additional information about the flow. The purpose of placing the pitot-static tubes in the rake is to determine: (1) the static head, (2) the flow direction, and (3) the flow speed, at location $G$ and $J$ in the boundary layer.

Our pitot-static tube design was based on the common single fluid probes design, i.e. the fluid in which the probe is operating is also the fluid used for pressure transmittal.

A pitot-static tube consists of two sets of ports - a total head port located at the nose of the probe and static port(s) located along the shaft of the probe. The design of these ports had to be altered from the single-fluid base line design to refiect the dual fluid system used here.

Static port. The static-port plays an important role in that it must determine the static head while under way. These static readings will not only be used with the pitot-static total head, but also with the total head pitot tubes. Several design questions concerning the static ports arose, such as the following: (1) the number of ports, (2) the size of the ports, and (3) the placement of the ports on the tube shaft.

The ports should be located far enough from the tip of the probe so that the flow is along the shaft of the probe, yet far enough forward of the rake so as to minimize interference effects with the rake. The static port is usually located a distance of three times the tube diameter aft of the probe nose. At the same time the tube length from the static port to rake strut is taken between eight and ten times the width of the rake strut.

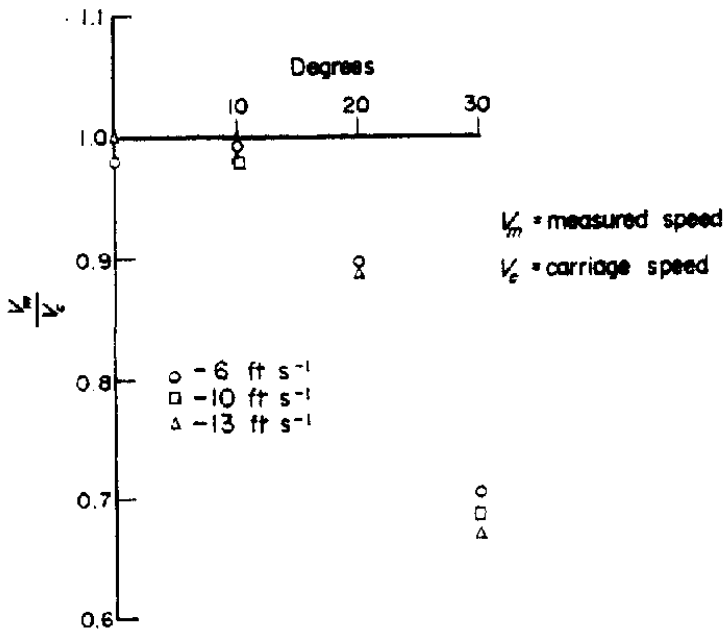

Fro. 7. The effect of flow angle on the pitot-static tube velocity measurements (total heed port). 

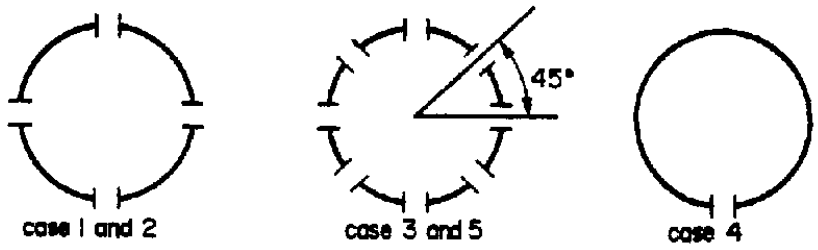

FIG. 8. Static port configuration for the pitot tube.

Several static port configurations were tested:

(1) 4 holes, in $x-y$ position, $1 / 32$ in. $(0.79 \mathrm{~mm})$ in diameter;

(2) 4 holes, in $x-y$ position, $1 / 16$ in. $(1.59 \mathrm{~mm})$ in diameter;

(3) 8 holes, in $x-y$ and $45^{\circ}$ position, $1 / 16$ in. $(1.59 \mathrm{~mm})$ in diameter;

(4) 1 hole, in the minus $y$ position, $1 / 16$ in. $(1.59 \mathrm{~mm})$ in diameter;

(5) 8 holes, in $x-y$ and $45^{\circ}$ position, $1 / 16 \mathrm{in}$. $(1.59 \mathrm{~mm})$ in diameter, with sand around tube shaft.

Figure 8 shows a cross section taken through the tube shaft in way of the static port.

The probe in the first configuration performed poorly, the air-water interface appeared to be at the static port, hence surface tension was significant.

Case two had the ports enlarged to $1 / 16 \mathrm{in} .(1.59 \mathrm{~mm})$ in diameter. Tube performance was significantly better.

Case three was tried in order to minimize sensitivity to cross flow or vortex flows.

Becanse of a time response problem associated with the breakdown of the surface film and with water entering the tube, a one static port tube was tried as ahown in case 4.

Open water performance of case 4 was about the same as for cases 2 and 3 . The response of probe types 2, 3, and 4 in position $J$ while under way is shown in Fig. 9. No one of the three deaigns seems superior to the other.

The effect of horizontal flow angle for case 4 can be seen in Fig. 10. The vertical flow angle is zero with the tube in rake position J. Note the speed dependence here. Combining the total head and static reading for the pitot-static tube produces the flow speed. In Fig. 11 one can see how this net speed changes with flow angle. An error of less than $4 \%$ exists to about $30^{\circ}$ with no apparent speed dependence for flow speeds above $10 \mathrm{ft} \mathrm{s}^{-1}\left(3 \mathrm{~ms}^{-1}\right)$.

As an additional test the pitot-static tube was moved from its position at the bottom of the rake (J) to a middle position $(G)$. At this location the static readings suffered an interference effect from the rake struts. We therefore suggest lengthening the tube by 1 in., moving the static port out away from the rake strut by 1 in.

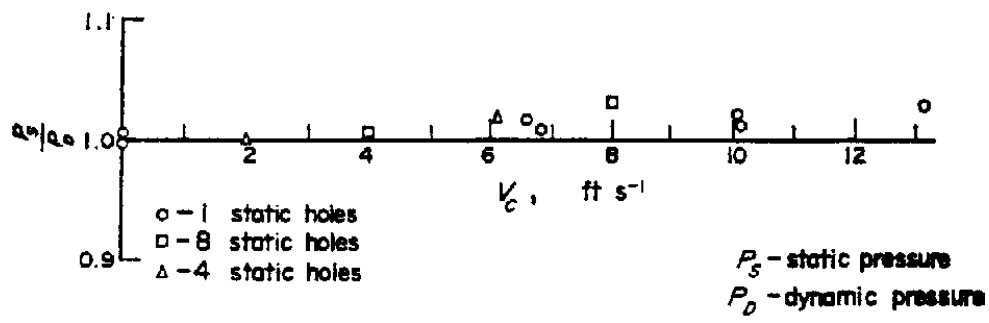

FiG. 9. Effect of speed on static port reading. 


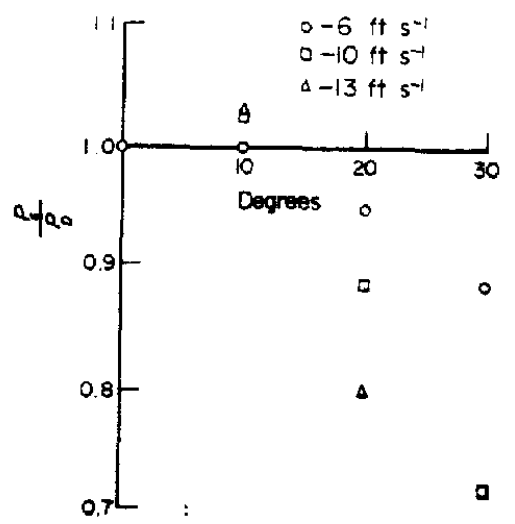

Fic. 10. Effect of flow angle on the static reading.

The performance of the total head pitot tubes has been outstanding, therefore it was only natural to try using one of these probes for a static reading. One total head pitot tube was mounted vertically open end down along the rake strut and tested.

For low speed applications this probe gave better results than the static reading from a pitot-static tube. At $13 \mathrm{ft} / \mathrm{s}^{-1}\left(3.97 \mathrm{~m} \mathrm{~s}^{-1}\right)$ the two probes gave comparable results. We have no data at higher speeds.

Total head port. The same considerations apply in the design of the total head port as for the total head pitot tube. The two important points are: (1) the realts of combining the static and total head readings must produce a flow apeed which is relntively insensitive to flow angies, and (2) the probe should not be 80 large that its presence alters its reading or the readings of its neighboring probes.

The first point requires a nose design that will produce preasure readings to match the static ports readings 80 as to produce a flow speed constant for at least $20^{\circ}$, the range of the total head tubes. The homispherical head such as that ued on a Prandtl tube produces a larger region of constant flow speed than, say, Brabbee's design which has a head similar to that on our total head tube, i.e. "open tube design".

Figure 7 shows the effect of incident flow angle on the total head reading for a hemispherical design nose. The resultant speed vs flow angle can be seen in Fig. 11 .

The hemispherical head does produce total head readings which are more directionally

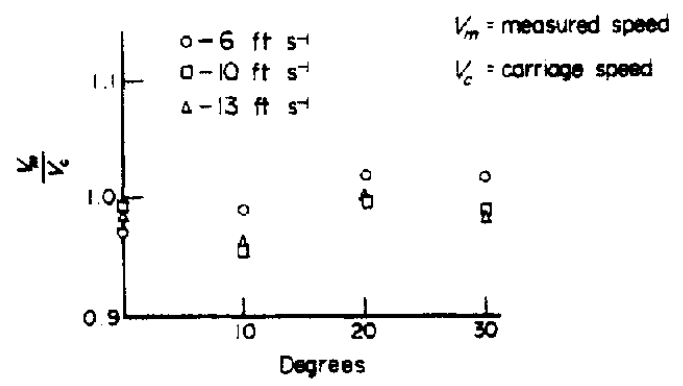

Fio. 11. Effect of flow angle on nensured speed. 
sensitive to flow than the total head tube design. This fact will be used to help determine flow direction.

To make the hemispherical head probe a reasonable size and still have a large enough port, the dimensions shown in Fig. 23b were selected.

\section{Interference effects}

Uniform stream. The first set of tests was designed to determine if one probe affected the performance of its neighboring probe. These tests were carried out by first removing all the probes from the rake except for one pitot tube. The test jig was then towed down the tank and the pitot tube results recorded. This was repeated several times with the pitot tube in a different location on the rake. All the tubes were then installed in the rake and towed. Comparison of the results for the individual probe at different locations and the results for all the probes in the rake in a uniform stream showed no interference effects on the total head readings. As reported earlier, interference effects between the static port on the pitot-static tube and the rake strut were detected.

Wake screen. The purpose of these tests was to determine how the probes would behave in a velocity gradient somewhat similar to that found in the ship's boundary layer. Because the boundary layer is a rotational flow field, interference effects between probes might show up.

In order to create a velocity gradient, a wake screen was constructed. The wake screen was made up of a wood frame $18 \times 18 \mathrm{in}$. $(460 \times 460 \mathrm{~mm})$ square with several layers of different density screens mounted on it. The first screen was made up of four layers of screen ranging from chicken wire to houschold window screen. The screen face was placed 12 in. $(300 \mathrm{~mm})$ in front of the pitot tube head. The velocity gradient produced here ranged from $0.85 V_{e}$, where $V_{c}$ is the uniform stream, at position $J$ (outermost position on the rake) to $0.4 V_{c}$ at position $\mathrm{B}$.

The wake screen was modified for a second series of tests. The trailing edge of the frame was faired and two additional layers of screen were added to the upper $6 \mathrm{in}$. (152 mm) of the wake screen. This produced a velocity profile which ranged from $0.85 V_{c}$ at position $\mathrm{J}$ to $0.3 V_{c}$ at position $B$. The tests were carried out using the same procedure as the uniform stream, i.e. removing all probes but one, then testing that single probe at different rake positions. This allows the velocity profile behind the wake screen to be constructed. No attempt was made to model a typical turbulent boundary layer with the wake screen. Next, all tubes were installed in the rake and the test was repeated. Comparison of the results shown in Figs. 12 and 13 indicate that there are some interference effects. Figure 12 shows interference effects at tubes $\mathrm{J}$ and $\mathrm{B}$; Fig. 13 shows a strong interference effect on tube $F$. This was probably due to the presence of the large pitot-static tube in position $G$.

\section{Determination of flow angle}

The incident fluid flow angle can be determined by taking advantage of the sensitivity of the total head port of the pitot-static tube to the flow angle. Assume an incident flow angle exists when the boundary layer rake is mounted in its zero position on the hull. Conduct the boundary layer test and record the readings from all probes. Plot the total head readings from the pitot-static tubes as a function of rake angle. Rotate the boundary layer rake to 


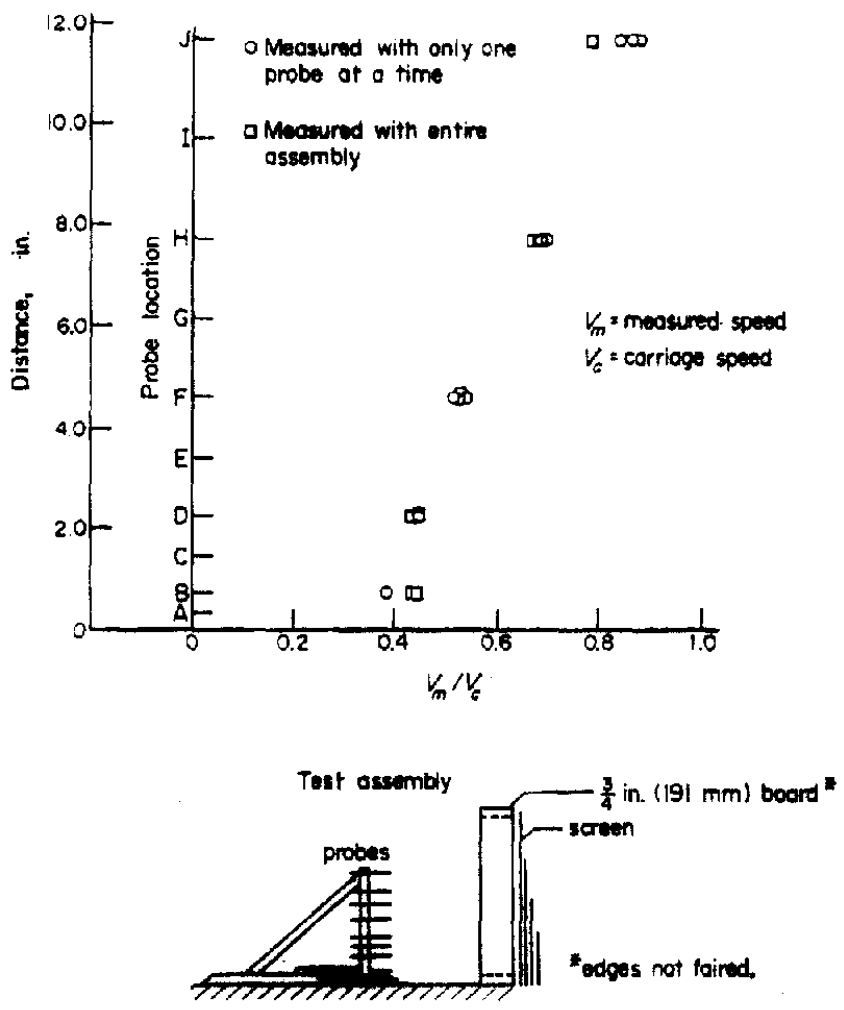

FIO. 12. Interference efrects of boundary layer device

the starboard and repeat the teat. Now rotate the boundary layer rake to the port and again repeat the test. More rake angles may be used if necessary.

Because the total head port reads a different value at some angle to the incident flow than at $0^{\circ}$ (see Fig. 7 , different total-head port readinga are observed. Of course, the actual total head has not changed. The resultant plot should have a maximum and this point becomes the incident flow angle. For example, see Fig. 14.

One of these plots will be produced for each pitot-static tube. If another pitot-static tube in the same rake produces a plot which yields a different incident flow angle it would imply that the flow direction is changing through the boundary layer, a phenomenon not unheard of in a rotational flow.

\section{INSTRUMENT PACKAGE FOR WAKE SURVEY}

The main goal of this research effort was to get reliable velocity profiles in the wake of a full-scale ship. As described earlier, it was decided to use five-hole pitot tubes in a manner similar to that described by Pien (1958). The major difference between our equipment and that of Pien (1958) was that the full-scale measurements were to be taken with air instead of water in the lines. This has been used successfully by Takahashi et al. (1970), among others. For a detailed drawing of the five-hole pitot tube and its mounting assembly, see Fig. 22. A photograph of the instrument is shown in Fig. 15.

The location of the holes on the $2 \mathrm{f}$ in. $(60.3 \mathrm{~mm})$ spherical head was as follows: one 


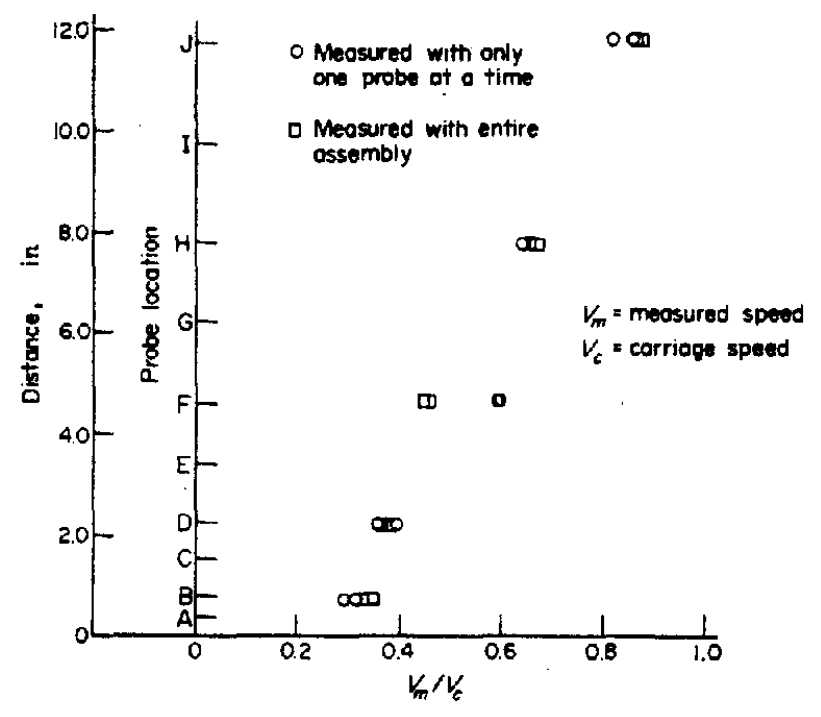

Test ossembly: Some as shown in Fig 12 except for the following:

(1) Edpes of $\frac{3}{4}$ in $(191 \mathrm{~mm} /$ boord foind

(2) More serien was odded, two pieces extending 6 in (152 $\mathrm{mm}$ ).

FiG. 13. Intarference effecte of boundary layer devico (with faired edpe).

hole to be placed on the centerline and the other four holes to be placed at an angle of $30^{\circ}$ (port and starboard, top and bottom) from the centerline. Figure 16 shows the placement of the holes. One benefit anticipated from this particular hole placement was that the presoure differences between outside holes and the center hole would be larger and thus less susceptible to scatter than the pressure differences for a hole placement that had the outeide holes nearer to the centerline. While this reasoning may be correct in principle, the fact that the fluid was viscous presented a number of problems. These problems and the steps taken to solve them are described below.

An attempt was made to calibrate the pitot tube assembly using the procedure outlined by Pien (1958). Pien states that within a region enclosed by a $40^{\circ}$ angular distance from the stagnation point, the pattern of pressure distribution is "nearly independent of Reynold's number". Unfortunately, our results indicated that for the range of pitot tube sizes being

$$
V=\text { measured speed }
$$

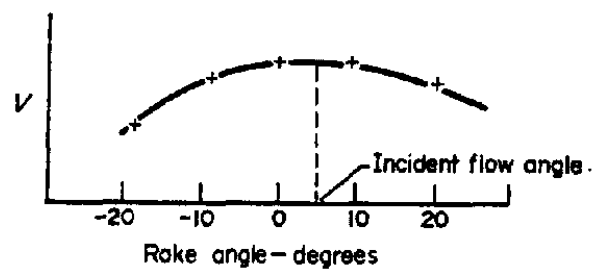

FiG. 14. Pitot static tubes total head readings plotted against rake angle. 


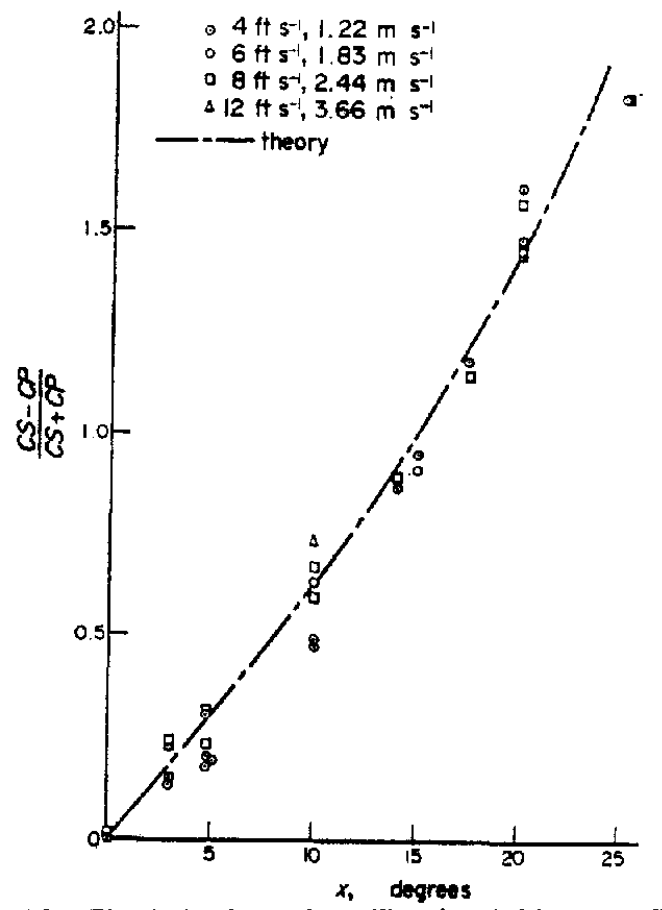

Fio. 17. Five-hole pitot tube calibration (without sand).

tested, a definite Reynold's number effect was present. This can be seen from Fig. 17 where the non-dimensional pressure coefficient $(-C S+C P) /(C S+C P)$ is plotted vo $X$, the ande in the horizontal plane between the incident flow and the centerline. Here CS and $C P$ represent the pressure differences between the center hole and the etarbourd hole, and the center hole and the port hole, respectively. A possible reacon for this variation may be deduced from a plot shown by Goldatein $(1965, \mathrm{p}$. 16). In that figure the drag conefieint $C_{D}$ of a sphere was plotted as a function of the Reynold's number Re. The drag coeficient is defined as the following:

$$
C_{D}=\frac{D}{\frac{1}{2} \rho U^{2}} d,
$$

where $D$ is the drag on the sphere, $\rho$ is the fluid density, $U$ is the free stream velocity and $d$ is the diameter of the sphere. The Reynold's number is defined as the following:

$$
\operatorname{Re}=\frac{U d}{v}
$$

where $v$ is the kinematic viscosity. One striking characteristic of the plot is the large drop in the drag coefficient at a Reynold's number of around $3 \times 10^{5}$. This is a well-known phenomenon and is due to the transition from laminar to turbulent flow. If a five-hole pitot tube were used in this range, its calibration curve would be speed dependent. For while the sharp drop in the drag coefficient is due to a large increase in pressure recovery over the after section of the sphere (this is a result of the point of flow separation moving aft), 
the pressure distribution over the forward part of sphere will also change. This is because the flow outside the boundary layer will more closely resemble that of a half-body when the Reynold's number is on the laminar side of the transition point. When the Reynold's number is on the turbulent side of the transition point, the flow will more closely resemble that of a sphere. The pitot tube described by Pien (1958) had a $1 / 2$ in. $(12.7 \mathrm{~mm})$ diameter ball size. When used in a towing tank at normal speed ranges of $5 \mathrm{ft} \mathrm{s}^{-1}\left(1.53 \mathrm{~m} \mathrm{~s}^{-1}\right)$ to $15 \mathrm{ft} \mathrm{s}^{-1}\left(4.58 \mathrm{~m} \mathrm{~s}^{-1}\right)$, the Reynold's number of the spherical head varies from $2 \times 10^{4}$ to $6 \times 10^{4}$, well below the critical Reynold's number of $3 \times 10^{5}$. The $2 \frac{8}{8}$ in. $(60.3 \mathrm{~mm})$ diameter ball will be used on a full-scale ship, encountering velocities ranging to $10 \mathrm{ft} \mathrm{s}^{-1}\left(3.06 \mathrm{~m} \mathrm{~s}^{-1}\right)$ to $30 \mathrm{ft} \mathrm{s}^{-1}\left(9.18 \mathrm{~m} \mathrm{~s}^{-1}\right)$. The Reynold's number corresponding to these flows will vary from $2 \times 10^{5}$ to $6 \times 10^{5}$. Thus the full-scale speed range will include speeds where there is a transition from laminar turbulent flow.

In an effort to solve the problem associated with the critical Reynold's number, fine sand granules were glued on the front of the sphere. Figure 18 shows a close-up of the pitot head. To keep the less firmly attached particles from washing off, a thin coat of lacquer was applied over the sand. The pitot tube was then tested and the results are shown in Fig. 19 where the non-dimensional pressure coefficient $(-C S+C P) /(C S+C P)$ is p.otted as a function of incident flow heading angle, $x$. It appears that the addition of the sand has made the pressure coefficient independent of Reynold's number, at least over the speed range tested. The dashed line in the figure is a theoretical curve based on the analysis done by Pien (1958).

A measure of the level of scatter with the sand attached was obtained by estimating the

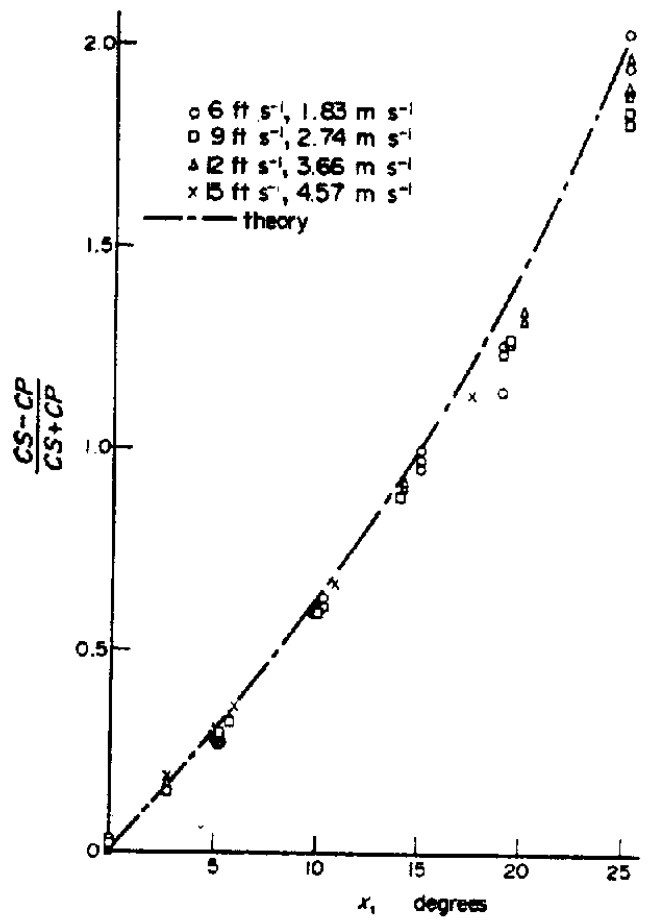

Fig. 19. Five-hole pitot tube calibration (with sand). 


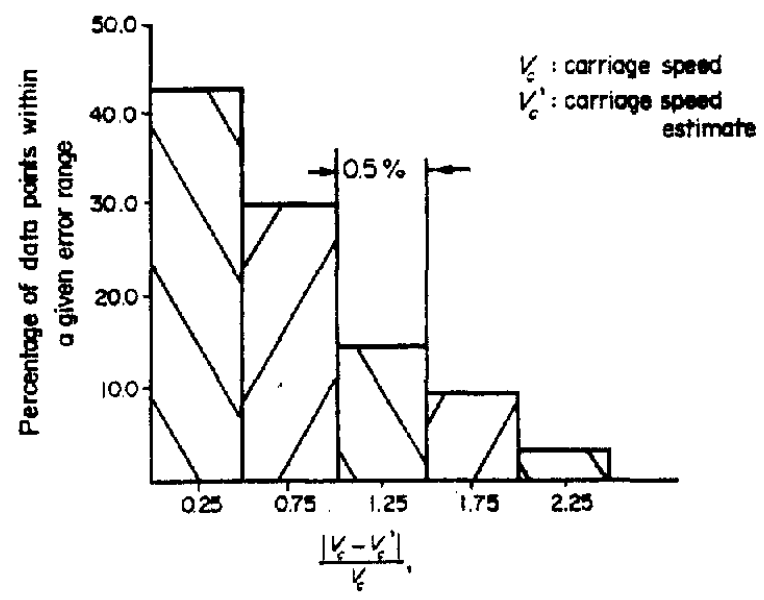

Fro. 20. Relative error in carriage speed prediction (based on 69 data points).

carriage speed and comparing that value with the actual speed. The procedure for finding the velocity components followed the method given by Pien (1958). A set of curves were faired through the data for values of $(C S-C P) /(C S+C P), C S / V_{h}^{2}$, etc. as a function of the incident flow angles. The actual pressure readings from the test were then used to enter the curves to estimate a heading angle and velocity components. The estimated carriage velocity based upon pitot tube measurements was then compared with the velocity from the carriage speed indicator. In Fig. 20, the relative number of times a data point occurred within a given error band is plotted against the relative error. As can be seen by the figure, more than $70 \%$ of the readings had a relative error of $1 \%$ or less.

Different types of coatings over the sand granules were applied to a test piece of brass. The piece was then placed in a salt bath for two weeks to see which coating worked best. Based upon that test, a coating composed of a thinned solution of epoxy glue was selected as having adequate properties.

Even though the tank tests indicated that a reasonable level of accuracy had been attained with the addition of sand on the pitot head, a recommendation to use this type of turbulence stimulation was not made. This was due to the following considerations:

(i) The calibration curves were very sensitive to the placement of sand around the tube holes. When one particle washed off, there was a corresponding change in the $C P / V^{2}$ curve. Different techniques were tried to ensure a secure bonding of the particles in the vicinity of the holes; some were successful. However, these proved to be very time consuming processes.

(ii) It was feared that in a salt water environment, the slime build-up on the epoxy coating would reduce the roughness of the pitot tubes thus negating any effect the sand particles might have.

(iii) Experience in wind tunnels has indicated that the amount of turbulence in the upstream flow has a marked effect on the drag coefficient of a sphere near its transition Reynold's number. For example, see Goldstein (1965). It was felt that there would be sufficient turbulence in the wake region of a ship to make the addition of sand a redundant measure. 

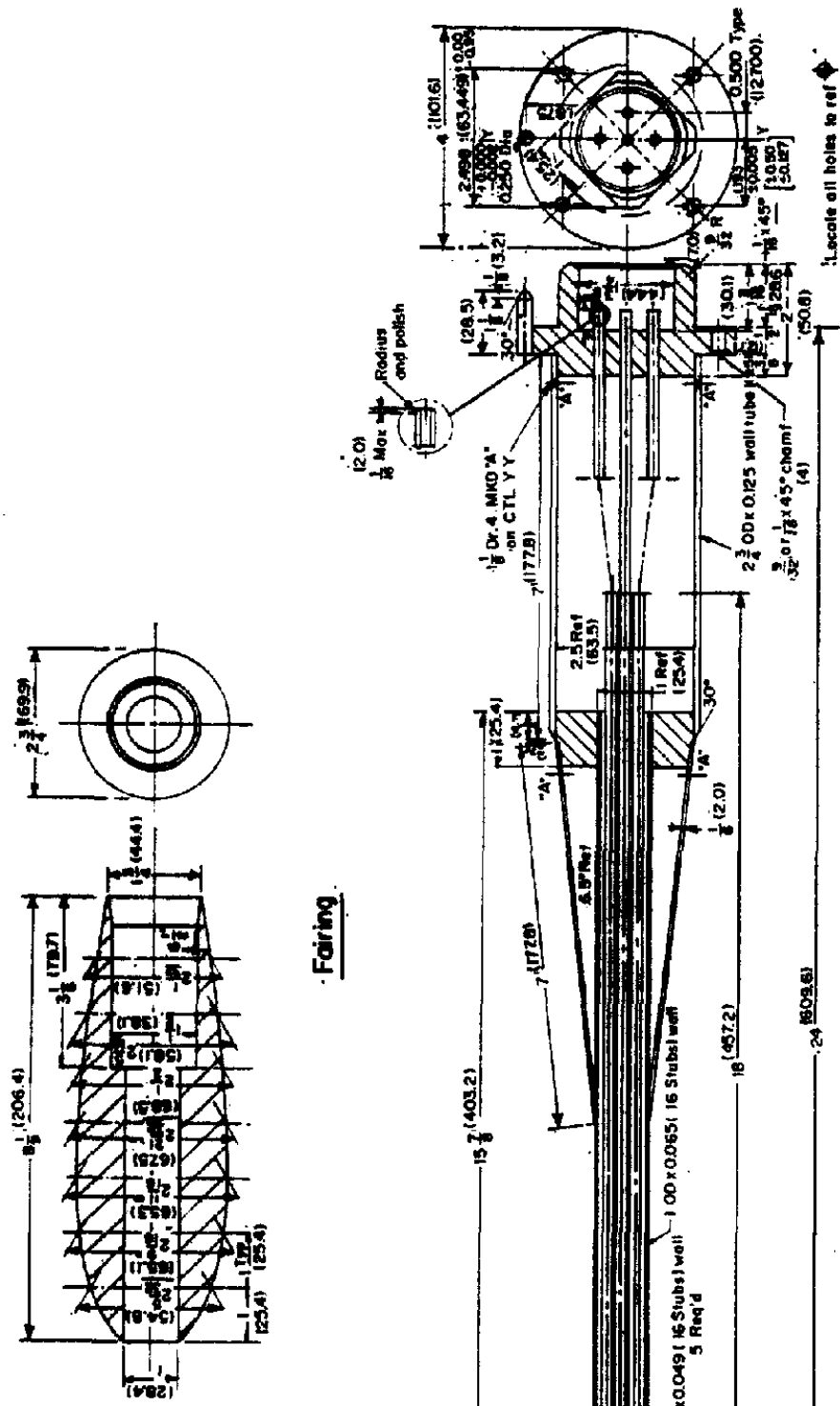

!
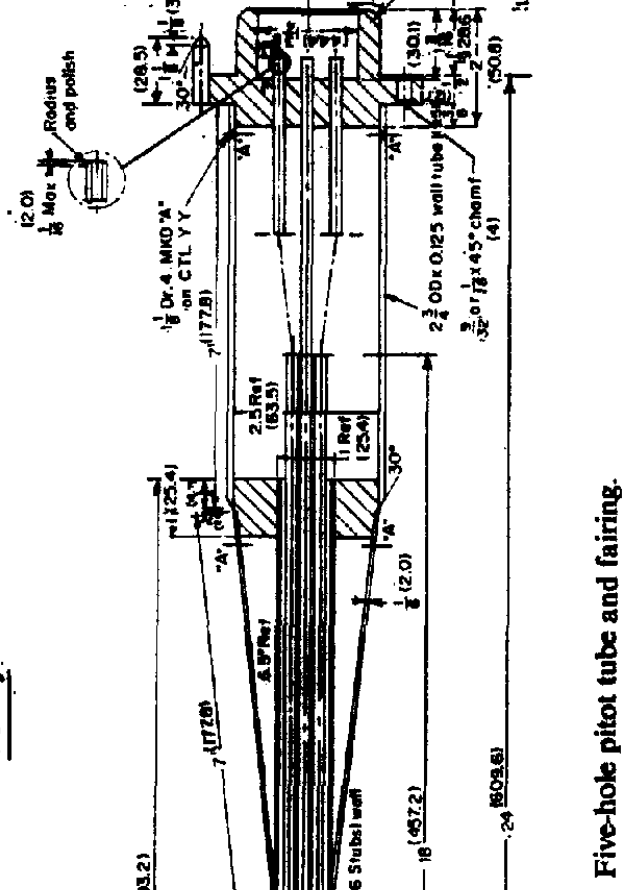

ถุำ

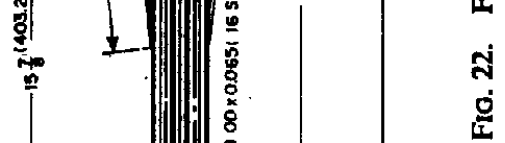


Takahashi et al. (1971) has suggested that a correction be made to account for the differences in head between the top hole, the center hole and the bottom hole. However,

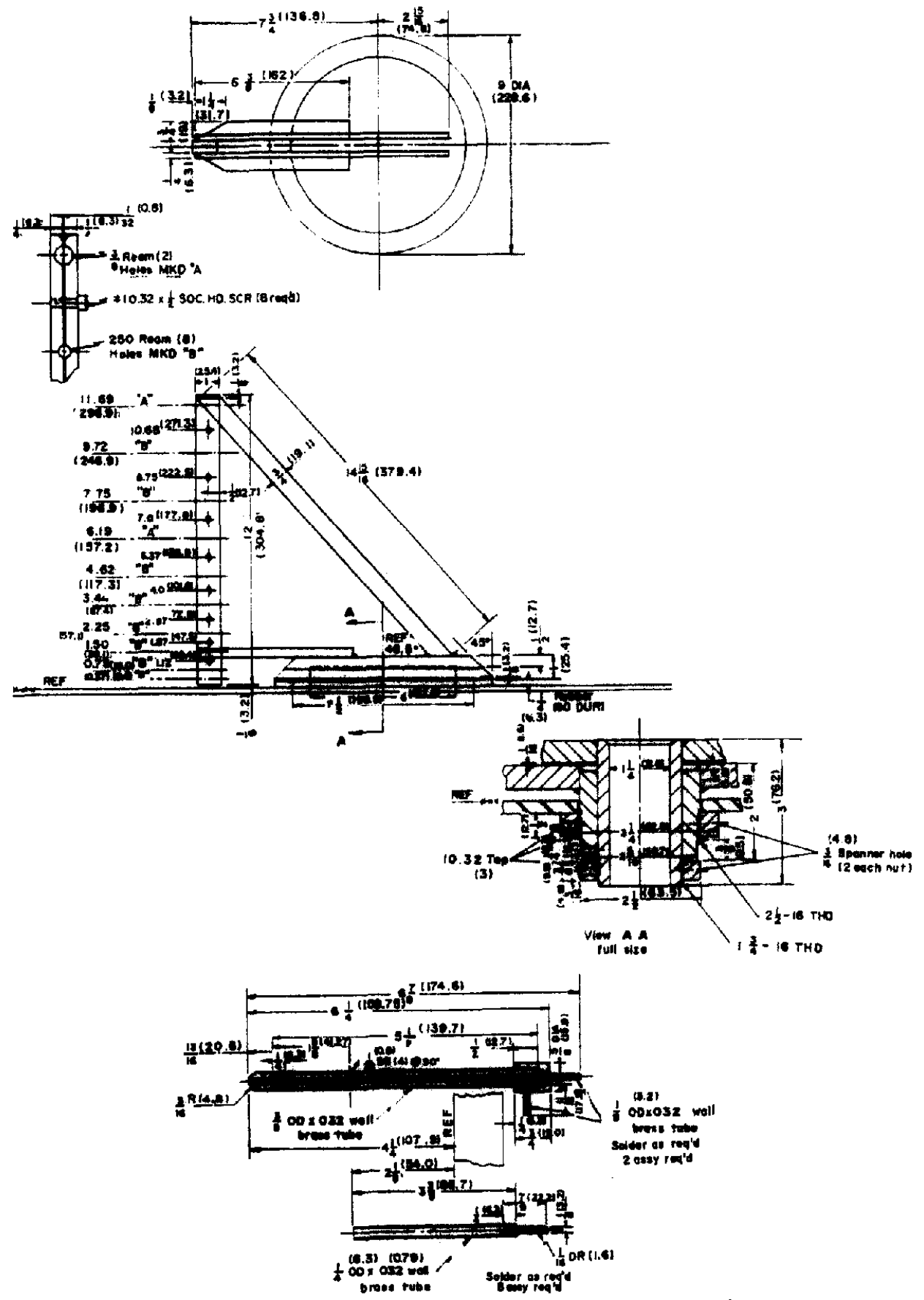

Fro. 23. (a) Boundary layer fixture; (b) Pitot-static and Total head pitot tubes. 
this means it would be necessary to locate the position of the air-water interface in the top tube. From visual observations, it seemed that the interface was very near to the surface of the sphere. Whether this would be true though for all speeds and all heading angies encountered we cannot say. When these differences in head were included in the calculations of the pressure coefficients, they were found to have a negligible effect for the range of speeds tested and were subsequently ignored.

The pitot tube assembly was also tested with a fairing attached to the stem as shown in

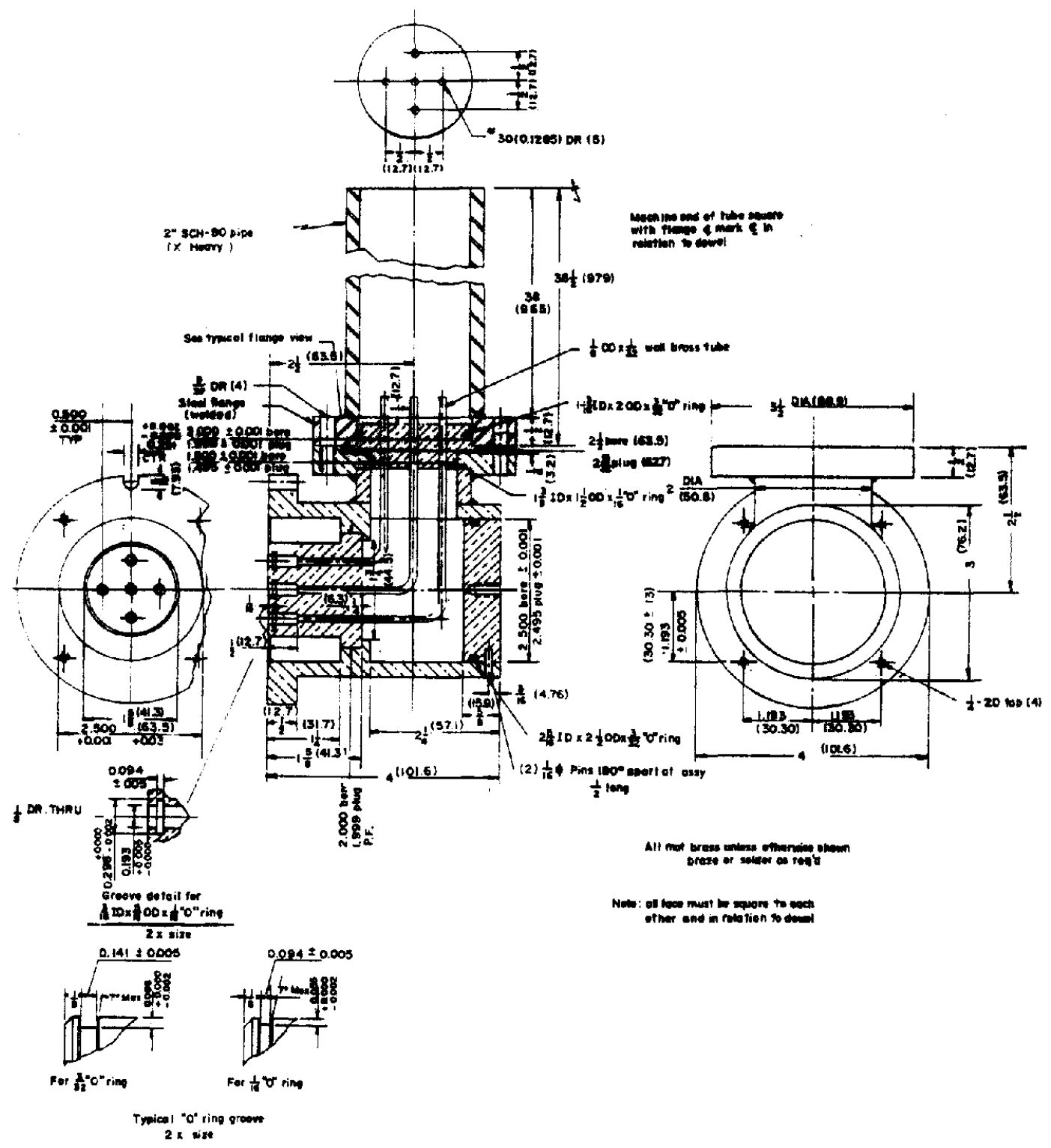

Fig. 24. Pitot tube adapter. 
Fig. 21. The purpose of this test was to determine whether the stem could be shortened. This would reduce any vibration problems associated with stem length. After a series of calibration runs, the results were compared with those from the pitot tube without the fairing and it was found that there was no significant difference. Thus the stem length could be shortened down to 3 in. $(7.62 \mathrm{~cm})$ without noticeably infuencing the pressure distribution.

Acknowledgement-This research was carried out under an Office of Naval Research contract, No. NOOO1476-C-1073, administered by DTNSRDC. We would also like to thank Professor R. KRLLER for his help in desiening and building the electrical components of this project.

\section{REFERENCES}

CANtun, H. J. S. 1974. Resistance, Propulsion and Wake Tests with HMS Penelope. R. Instn Naval Architocts, Spring Meeting.

Executive Committe for the Project of Ship Boundary Layer Measurements. 1971. Measurement of Boundary Layer of Shipa. Reports Res. Inst. appl. Mech., Kyushu Univ. XIX, No. 63.

Gouderesw, S. 1965. Modem Dewilommuts in Fludd Dymamics. Dover, Now York.

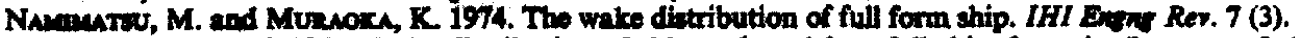

Nammurev, M. ot al. 1973. Wake distribution of ship and model on full ship form in Japanese. J. Soc. Navel Arelthects Japen 134.

Prev, P. C. 1958. The Fivo-Hole Spherical Pitot Tube. DTMB Rep. 1229.

TAkuruen, H. af al. 1970. Menrurement of Velocity Distribution Ahead the Propelier Diak of the Ship (in Inpaneoo). J. Soc. Navel Areblicets West Japan 123.

TAnoucan K. and Furte, T. 1970. Comparison of Velocity Distribution in the Boundary Layer Between Ship and Modeh, J. Soc. Naval Archifteets Jaman 127.

Yoroo, K. 1974. Meanurement of Full Soale Wako Conracteristics and Their Prodietion from Model

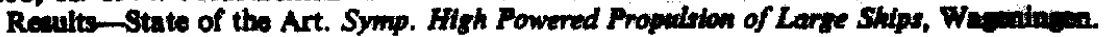

